\title{
Investigation of rain and haze attenuations impact on proposed SCM-SAC-OCDMA-FSO system with optical amplifier
}

\author{
Hashem A. Jabar ${ }^{1 *}$, Rshid A. Fayadh ${ }^{2}$ and Mariam M. Abud ${ }^{3}$ \\ Research Scholar, Middle Technical University, College of Electrical Engineering Techniques, Baghdad, Iraq ${ }^{1}$ \\ Assistant Professor, Department of Electrical Engineering, Middle Technical University, College of Electrical \\ Engineering Techniques, Baghdad, Iraq ${ }^{2}$ \\ Assistant Professor, Department of Physics, Al-Mustansryia University, College of Education, Department of \\ Physics, Baghdad, Iraq $^{3}$
}

Received: 01-July-2018; Revised: 03-November-2018; Accepted: 12-November-2018 (C)2018 ACCENTS

\begin{abstract}
Free space optics (FSO) is a new modern communication technique, which allows users to transmit their data in secure state where line-of-sight (LOS) mechanism used to ensure successful transmission of optical laser signal through free space. While optical laser beam travel through unguided medium where no fixed medium allocate to each user to transmit its data, so large atmosphere phenomena like (rain, fog/haze, etc.) can effect on the transmission signal. Over the years, many techniques were implemented in system design of FSO to reduce the effect of multiple access interference (MAI) and weather phenomena on the optical laser beam attenuation. Therefore, this study proposed sub-carrier multiplexing (SCM)-spectral-amplitude coding (SAC)-optical code division multiple access (OCDMA) FSO system with operational amplifier to reduce the effect of rain and haze on laser beam attenuations. This study, conducted in three different regions of Iraqi cities, which are Baghdad, Basra, and Mosul. The FSO system proposed consists of the combination of three techniques. OCDMA technique start using in system design to support a large number of users that share the same single laser beam and provide multiple access to the system. SCM and SAC also used to decrease the effect of MAI on the signal quality to decrease the external effect and increase network capability. Type of code used in the system called multi-diagonal (MD) code to support better in signal quality, providing zero cross correlation even if the number of user increase in the system. An additional component added to the system in order to enhance the signal quality called "optical amplifier" that used to add more energy to the signal to allow user signal travels through long distance. Based on these techniques, signal can travel through the rain and the haze weather state in three different regions of Iraqi cities [BAGHDAD, BASRA and MOSUL] which are with less bit error rate (BER) in final point and acceptable distance (reach to $1.35 \mathrm{~km}$ in rain state and $1.85 \mathrm{~km}$ in haze weather state). In addition to that, this paper shows a new laboratory method to study the effect of free space particles (rain and haze) on laser signal by using the glass room that must have the ability to perform rain and haze weather effect. Then, using an ocean optic device to collect the light from the room and translate it to the PC. The practical part ensures that rain largely attenuate wireless signal than haze particles. The results of practical part indicate the relation between the intensity count of light in the receiver side and the different types of wavelength $(670 \mathrm{~nm}, 785 \mathrm{~nm})$ that used in the laboratory experiment.
\end{abstract}

\section{Keywords}

Free space optic, Multiple access interference (MAI), OCDMA, Multi-diagonal code, SCM, SAC, Optical amplifier.

\section{Introduction}

Nowadays, the communication domain and network topology in all the world increase to serve human requirements and needs in different connection paces, such as ship to ship and temporally connection that serves temporal function.

\footnotetext{
*Author for correspondence
}

FSO come to replace the use fiber optic cable in some places of communication in which it is difficult or impossible to extend cable due to the harsh environment or having river problem. FSO are used to provide point-to-point connection (when need large amounts of bandwidth) or point to multi point (less bandwidth than point-to-point) [1].

The main FSO channel losses are due to absorption and scattering process, which is form the total atmospheric attenuation. This process due to various 
scattering particles presents in the atmosphere channel like water, carbon dioxide, haze, fog, etc. that attenuate carrier power and lead to decrease signal performance. Scattering and absorption are not only attenuating the signal beam in the atmosphere, but it is the primary cause for sky radiance, which introduce noise in daytime communication. In 2007, Sasaki et al. [2] introduced a first optical code division multiple access transmission, data propagate through 150 meters only. The fiber cable mixed with optical wireless because of short distance optical wireless can cut it. OCDMA-FSO communication plays an effective rule in the proposed system that provide high rate of data transmission for each user with less particles weather absorption [3].

SAC technique starts using with OCDMA to support the increment in users that OCDMA permitted in the system of FSO, it used to limit the effect of multipleuser-interference (MUI) with supporting MD code. Consequentially, MD code has zero cross correlation value even when the number of user increase in the system, zero correlation, probability in the system ensure that user data does not interaction between each other through the unguided medium [4]. SCM technique also used in FSO system to multiply user data electrically with different carrier frequency and then modulated it with the laser beam using external optical modulator (EOM) then initiate transmission towards the receiver. In order to add more enhancements to the FSO system, optical amplifier that act as intermediate stage are added to the system of SCM-SAC-OCDMA to compensate the energy that signal losses when propagate through the unguided medium. The number of the amplification process on the FSO signal is according to the gain value, which is varying between (1-100) $\mathrm{dB}$. This paper characterizes the optical attenuation of FSO communication systems in Iraq based on the weather patterns in three different regions (BAGHDAD, BASRA and MOSUL) and determine the atmospheric attenuation of FSO Communication systems in Iraq. This paper proposed techniques that are combined in one system known as SCM-SACOCDMA with FSO based on MD code and intermediate stages to find the effect of rain and haze particles on signal quality. These systems are built using "opts software" Ver. 7, also this study shows how can build FSO system in laboratory experiments to notice the effect of rain and haze particles on signal quality. Figure 1 shows the concept of FSO technique that used to connect building to building in LOS between the two points.

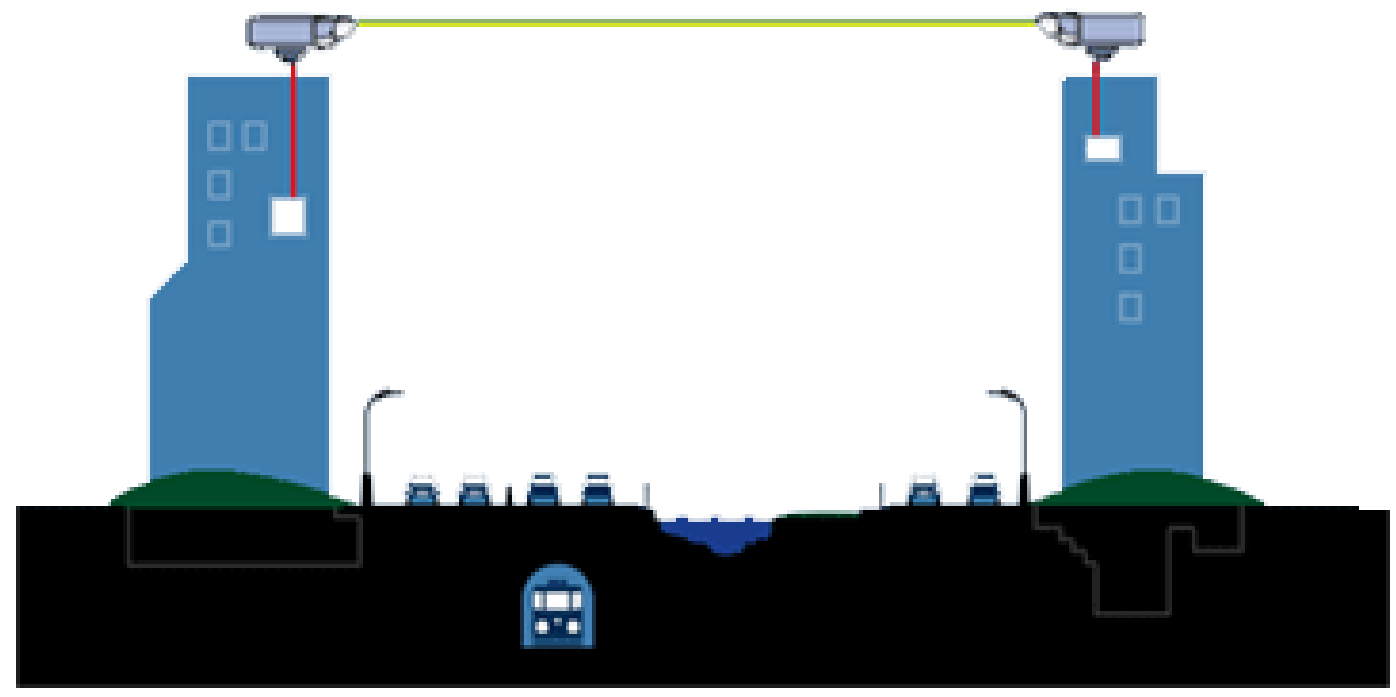

Figure 1 FSO used as connection techniques in different applications

\section{Materials and methods}

Figure 2 shows the block diagram of SCM-SACOCDMA based on MD code. MD code essentially used in the proposed system more than other code like random diagonal (RD) code or diagonal double weight (DDW) code because it is the only type of code in FSO that ensure zero interaction probability even when many users share the same system. System of SCM-SAC-OCDMA contains the following parameters: In the transmission side, data with independent unipolar digital signal is optically modulated onto the code sequence using an optical 
Jabar et al.

external modulator (OEM) .The external modulator that used in the system called Mach-Zehnder modulator (MZM) that used to modulate the electric data " users information " with the laser beam (which act as the main carrier of FSO) and then initiate transmission through the air. The laser that used in the proposed system called continues wave $(\mathrm{CW})$ laser to allow the laser to continue emitted through free space to provide continuous service to transfer user data to the final destination. Due to no guided link in optical transmission and no fixed link that user's data can flow through it, a large attenuation occurs when signals propagate through free space and different weather phenomena occur. In order to get best signal quality and minimum BER in receiving side, the optical amplifier was used to compensate the loss in signal power and capability to transmit data through long distance with less BER [5]. In the receiving side, de-multiplexing technique act as decoding to decode the MD code and decompress user data and then separate the optical signals and passes it to fiber Bragg grating (FBG) filter to reject the unwanted light spectrum. The signal then passed to the avalanched photo-detector (APD) to collect the light and convert if form light form to electric form. The electric signal after passed through the APD entered through a different filter (low pass filter "LPF") and (band pass filter "BPF") to reject the unwanted signals and passes the required signal to the receiver sides. Figure 2 shows how the component of the proposed system is arranged to present the complete system that must building using "opt system" that offer a strong base to interact with many components.

To implement the SCM-SAC-OCDMA system with optical amplifier, parameter in Table 1 must be taken to get the result of each weather effect by software implementation:

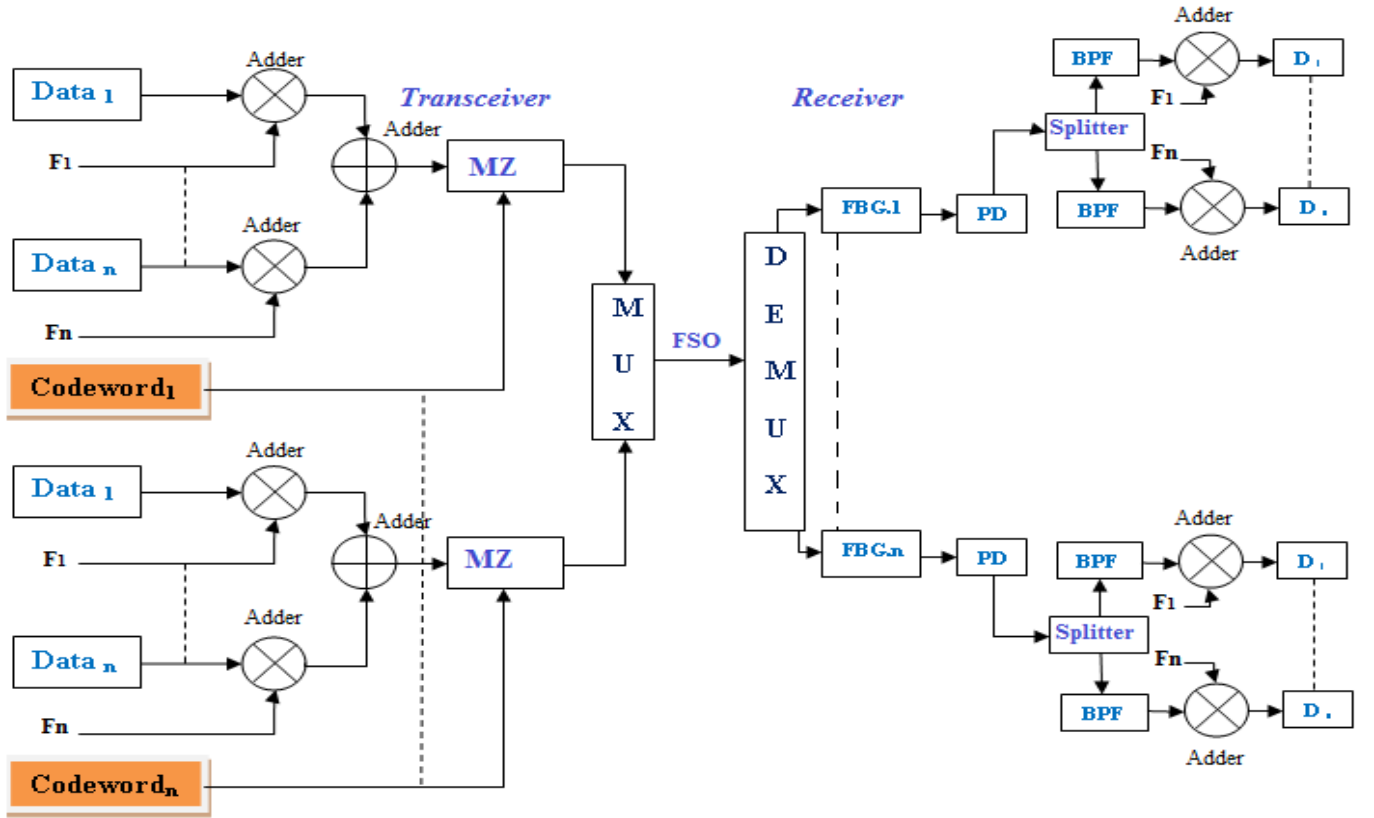

Figure 2 Proposed system SCM-SAC-OCDMA

Table 1 Parameter used in the proposed system

\begin{tabular}{llllll}
\hline Sym. & Parameter & Value & Sym. & Parameter & Value \\
\hline Psr & Broadband power & $5 \mathrm{~dB}$ & $N_{S}$ & No. of subcarrier & $2-20$ \\
\hline$\propto$ & Rain or haze drop intensity & Table $(1) \&(2)$ & $\mathrm{G}$ & Gain of amplifier & $30 \mathrm{~dB}$ \\
\hline $\mathrm{B}$ & Electrical B.W & $311 \mathrm{MHz}$ & $f_{n o}$ & Noise figure of op.amplifier & $1.9 \mathrm{~dB}$ \\
\hline$T_{n}$ & Noise temperature & $300 \mathrm{Kelvin}(\mathrm{K})$ & $\mathrm{hv}$ & Photon energy & $1.28 \times 10^{-19}$ \\
\hline $\mathrm{e}$ & Electric charge & $1.6 \times 10^{-19}$ coulomb $(\mathrm{c})$ & $B_{o p}$ & Bandwidth of op. amplifier & $200 \mathrm{THz}$ \\
\hline $\mathrm{h}$ & Planck's constant & $6.66 \times 10^{-34} j$ oul second & $\mathrm{R}$ & Responsively of photo detector & $1.32 \times 10^{13}$ \\
\hline$k_{b}$ & Boltzman's constant & $1.38 \times 10^{-23} j / k$ & ---- & Optical power o/p & $4.55 \times 10^{-3} \mathrm{Watt}$ \\
\hline
\end{tabular}




\section{Performance analysis of SCM-SAC- OCDMA FOS system based on rain and haze attenuation}

Rain is one of the most important phenomena that have a distance-reducing impact on the optical wireless signal. The rain particles size can vary between $(200$ to $2000 \mu \mathrm{m}$ ), the rain particles absorb the photon energy and capable of attenuation laser power when travel through free space [6].

The rain attenuation for each rain dropping value can calculate using the following expression [7]:

$\propto_{\text {rain }}=1.076 \times \mathrm{R}^{0.67}$

Where $\mathrm{R}$ is the rain intensity rate in $(\mathrm{mm} / \mathrm{hr})$ and the rain rate of Iraqi cities with different rain intensity effect in the three different regions [BAGHDAD, BASRAH and MOSUL] is shown in Table 2 [8]:

Table 2 Rain rate intensity value in IRAQ, year 2017

\begin{tabular}{lccc}
\hline Region & $\begin{array}{c}\mathbf{R} \text { at heavy } \\
\text { rain }(\mathbf{m m} / \mathbf{h r})\end{array}$ & $\begin{array}{c}\mathbf{R} \text { at } \mathbf{~ m i d} \text { rain } \\
(\mathbf{m m} / \mathbf{h r})\end{array}$ & $\begin{array}{c}\mathbf{R} \text { at light } \\
\text { rain } \\
(\mathbf{m m} / \mathbf{h r})\end{array}$ \\
\hline Baghdad & 30.3 & 11.7 & 3.8 \\
Mosul & 78.0 & 45.7 & 2.1 \\
Basrah & 26.1 & 13.0 & 1.4 \\
\hline
\end{tabular}

The second weather phenomena that start to widely occur in free space "air" especially in the Middle East region is haze weather phenomena where dust particles are separate through the propagation path of the laser beam that hold information signal. The dust leads to decrease the visibility of the weather and effect on the signal quality by scattered the laser light and cause losses in the user data because the receiver aperture diameter cannot be able to collect the entire laser light, so it will lead to lost in user data. The haze attenuation value in $(\mathrm{dB})$ is less than rain weather attenuation because its particles are smaller than water drop particles [9]. Simple formulas to calculate fog/haze attenuation is shown in Equation 2 [10] [11]:

$\propto=\frac{3.91}{\mathrm{~V}}\left(\frac{\mathrm{W}}{550}\right)^{-\mathrm{q}}$

Where $\mathrm{q}$ is the size distribution coefficient of scattering, $\mathrm{V}$ is visibility range (in Kilometre) that shown in Table 3 and $\mathrm{W}$ is the wavelength (in nanometre) that used in the system of SCM-SACOCDMA. According to Kruse model, $\mathrm{q}$ is given as:
International Journal of Advanced Computer Research, Vol 8(39)

$$
\mathrm{q}= \begin{cases}1.6 & \mathrm{v}>50 \\ 1.3 & 6<\mathrm{v}<50 \\ 0.585 \times v^{\frac{1}{3}} & \mathrm{v}<6\end{cases}
$$

Table 3 The minimum visibility range in three different regions of Iraqi cities

\begin{tabular}{llll}
\hline Month & January & March & September \\
\hline V.in Baghdad $(\mathrm{km})$ & 0.2 & 2 & 10 \\
V.in Basra $(\mathrm{km})$ & 0.8 & 2 & 10 \\
V.in Mosul $(\mathrm{km})$ & 0.2 & 3 & 6 \\
\hline
\end{tabular}

Now, the performance of the SCM-SAC-OCDMA system based on FSO is greatly affected by weather conditions, poor weather conditions cause low signalto-noise ratio (SNR) value and an increased bit error rate (BER). SNR is ratios that find the relation between the information signal and the background noise and when the SNR increase BER constantly decrease and provide the system to be more efficient. The effect of thermal noise and shot noise and intermodulation distortion in the photo detector, as well as the effect of rain or fog/haze attenuation $\left[\alpha_{\text {spec }}(\lambda)\right]$ on the signal power received $\left(\mathrm{P}_{\mathrm{sr}}\right)$ must take its effect when calculates the SNR value. The SNR and BER are calculated by using a Gaussian approximation equation to find the system performance of the proposed system.

The following Equations 5 and 6 are used to find the SNR \& BER of SCM-SAC-OCDMA with optical amplifier:

$\mathrm{SNR}=\left[\mathrm{I}^{2} / \sigma^{2}\right]$

Where $\mathrm{I}^{2}$ is the average signal power, $\sigma^{2}$ is the average noise powers in which their values are calculated using the following Equation:

$$
\sigma^{2}=I_{s h}^{2}+I_{t h}^{2}+I_{I M D}^{2}+\alpha_{s p a c e}
$$

Then, total SNR are calculated using Equation (5)

$\mathrm{SNR}=\frac{\left[\frac{R P s r W}{N} u n, k m n, k\right]^{2}+\text { optical power o.p. }}{\frac{e B R p_{s r} w}{N}+\frac{4 k_{b} T B}{R l}+P^{2} s r R^{2} m n, k\left[\frac{D 1,1,1}{32}+\frac{D 2,1}{64}\right]+\alpha+(\mathrm{G}-1) \mathrm{f}_{\mathrm{no}} \mathrm{h}_{\mathrm{v}} \mathrm{b}_{\mathrm{op}}}$

Where $\alpha$ shows the attenuation in Equation 1and 2 . To get the results of BER for each SNR value, the following Equation 5 used to find BER value:

$\mathrm{BER}=\frac{1}{2} \operatorname{erfc} \sqrt{\frac{S N R}{8}}$ 
Jabar et al.

\section{Results and discussions}

4.1Software implementation

The SCM-SAC-OCDMA systems with optical amplifier are built using 'opts system' software version 7 to see the effect of rain and haze on the performance of FSO and the parameters that used to build the system are obtained in part 2 with the block diagram of the proposed system. The value of wavelength is $1550 \mathrm{~nm}$ that used in the system rather than $1330 \mathrm{~nm}$ or $785 \mathrm{~nm}$, because $1550 \mathrm{~nm}$ is less affected by the weather particles and the short wavelength always scattered in free space more than long wavelength and the long wavelength is always best choice in communication system. Any wavelength that is shorter than $800 \mathrm{~nm}$ is unused in wireless communication technique because attenuation due to Rayleigh scattering is high. Figure 3 shows why wavelength of $1550 \mathrm{~nm}$ is preferred to be used in FSO technology.

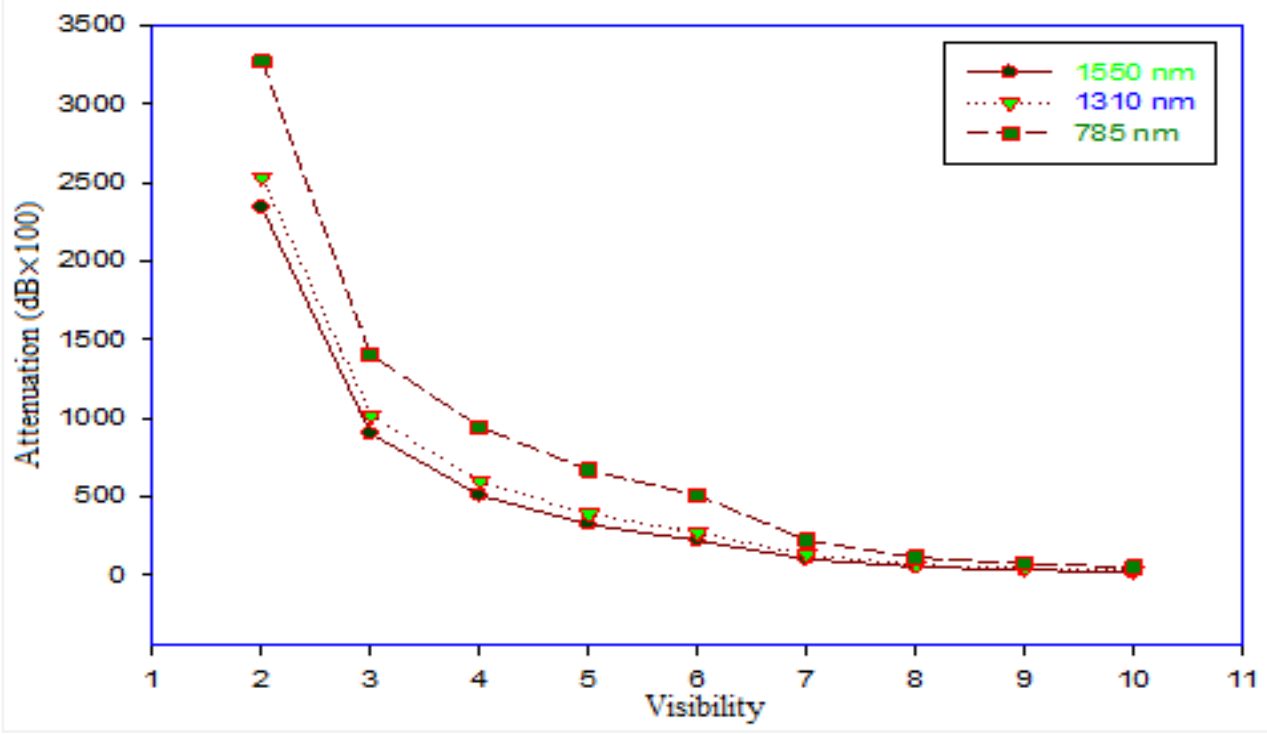

Figure 3 Graph shows that $1550 \mathrm{~nm}$ has minimum attenuation

The rain weather state has largely effect on the performances of the signal because rain particles are larger than dust particles and hence lead to absorb the photon energy more than dust or water vapour particles and increase BER on the receiving side due to decrease in SNR value. Increases in rain dropping (rain particles reaches to $0.1 \mathrm{~cm}$ in heavy rain) will increase in BER and decrease the distance between the transceivers because a large number of water particles are separated through the free space which laser light use it to deliver the user data. In addition to that, the Heavy rain lead to block the transmission path more than mid and minimum rain falling state. Figure 4 shows the attenuation of rain state at different conditions [light, mid, high].

Figure 5 shows the optical spectrum analyzer of the SCM-SAC-OCDMA and how can it affect when laser beam that hold user data transfer without any guided media through different weather phenomena. The water particles lead to decrease the system power (by absorbing the signal energy) hence led to block the signal from reaching to the receiver point and decrease system characteristic. The transmission spectrum shows the normal range of signal power that ranged between $(-100$ to -2$) \mathrm{dB}$ in which the signal is not affected by the weather or other external effect. While in receiving side (Figure $5 \mathrm{~B}$ ) where the signal affected by large rain particles and the range of receiving power decreased and reaches between $(-100$ to -20$) \mathrm{dB}$. It means that the receiving signal decrease due to absorption by the weather particles.

In the presence of rain weather particles, a system can travel through $1.35 \mathrm{~km}$ as a transmission distance and each user can send 1 Gbps under heavy rain. BER for heavy rain $1 \times 10^{-14}$ and this value decreases when rain moderately between mid and drizzle rain because the external effect is less than in heavy rainy weather. The proposed system shows better performance when compared with the latest study of FSO by Fayadh and Hamadi in 2016 [12] which Sub carrier multiplexing technique has been used in FSO system with help of SAC-OCDMA under rain 
weather states. The proposed system transmits data with $155 \mathrm{Mbps}$ for each user share the system with minimum BER reach to $10^{-9}$ under strong weather phenomena. In the presence of fog/haze, signal performance should be record better result than rain because haze attenuation is less than rain. Figure 6,7 and 8 shows the eye diagrams which are image that look like the opening of a human eye and it's a common indicator of the quality of signal in highspeed digital transmission when travels from its point to its final destination. It is clear that pure eye means the signal travels in a smooth path with minimum weather effect. The visibility range that is less than (200 meter) and according to Equation 2, the attenuation in $\mathrm{dB}$ record only 13 . This attenuation leads to increase in BER and the eye diagram seems to be in unregulated shape. Visibility range that is higher than $(10 \mathrm{~km})$ means less particle dust separated in the air. Figures 6, 7 and 8 shows the eye diagram of three ranges of visibility in Iraq [minimum visibility $(>200 \mathrm{~m})$, mid visibility $(1 \mathrm{~km}<\mathrm{v}<9 \mathrm{~km})$, high visibility $(>10 \mathrm{~km})]$. The haze particles decrease the visibility rang of the transmission path and scattered the signal laser beam, hence the receiver aperture diameter not able to collect all the transmitted information. In haze weather, BER for minimum visibility records is $1 \times 10^{-15}$. Finally, in hazy weather, signal can propagate through $1.85 \mathrm{~km}$ as transmission distance and each user send $1 \mathrm{Gbps}$ with transmission power $5 \mathrm{~dB}$ only.

According to the result of the two-weather state, the signal can cut acceptable distance with the help of optical amplifier and all the techniques used to form FSO system with the ability in reducing the effect of weather phenomena and other external effect that signal may collide through the propagation path.

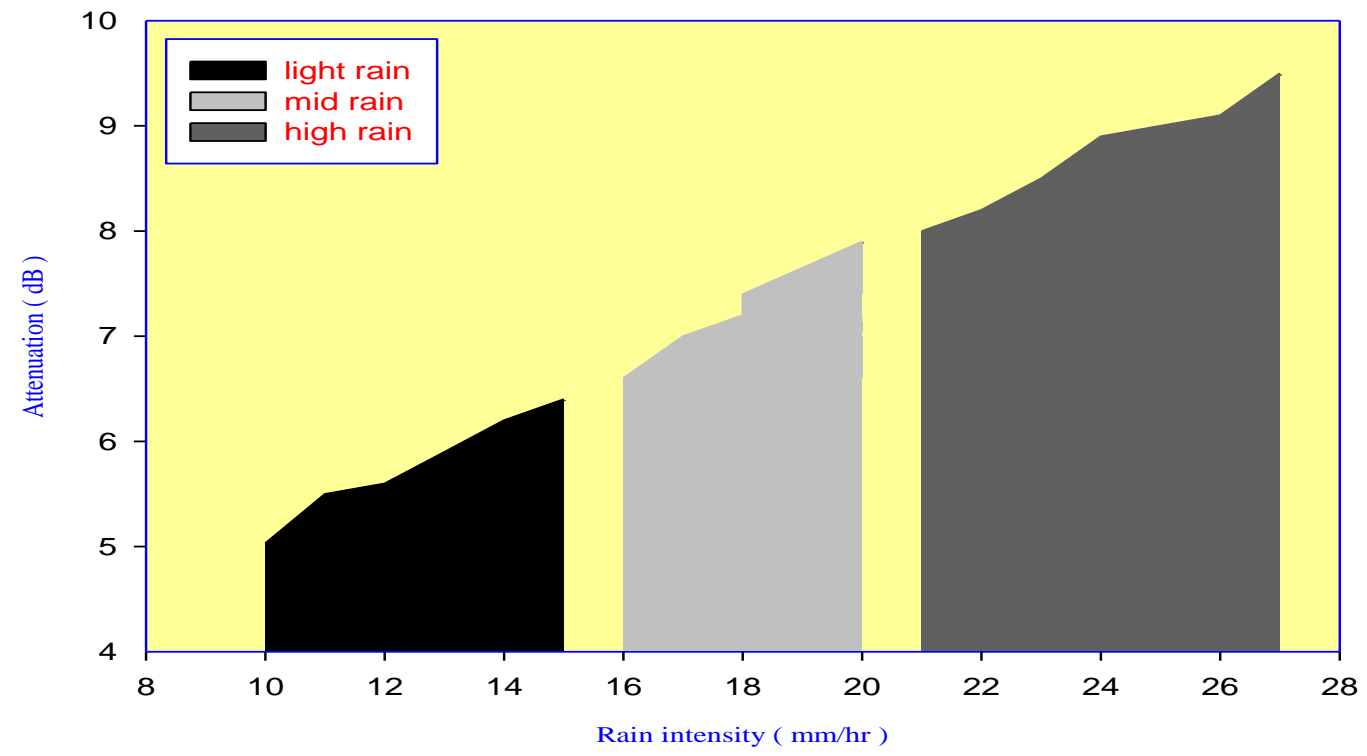

Figure 4 Attenuation of rain state in different intensity count 
Jabar et al.

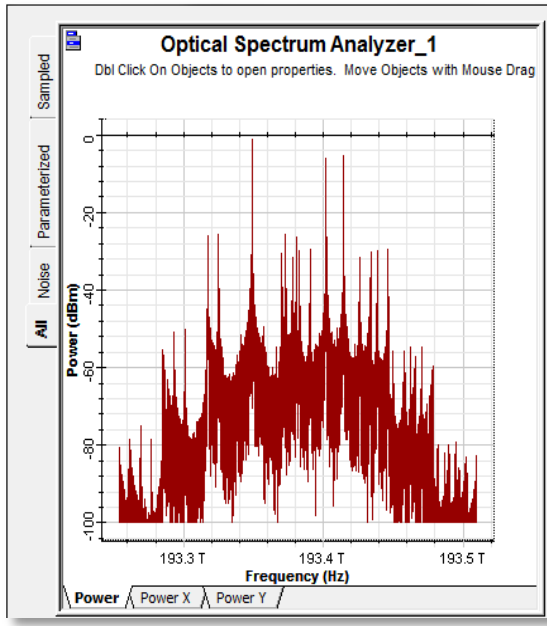

A

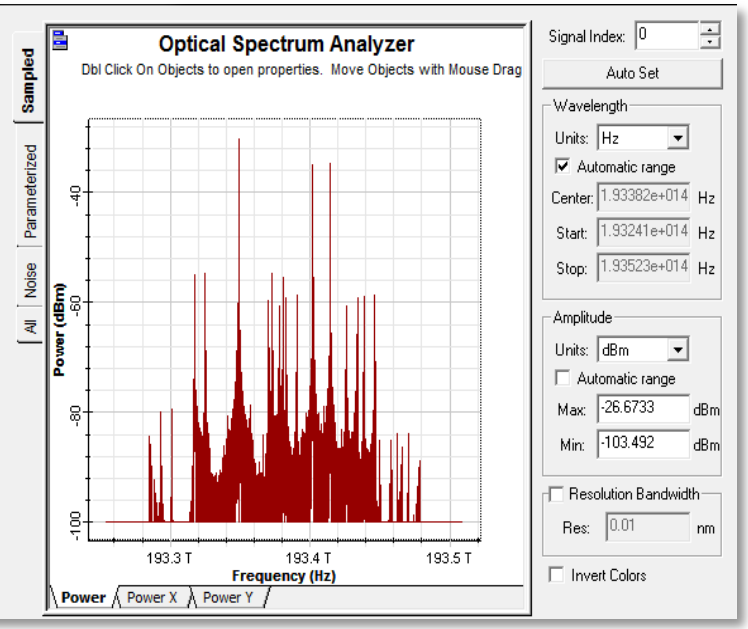

B

Figure 5 The power spectrum (A) before transmission and (B) after transmission
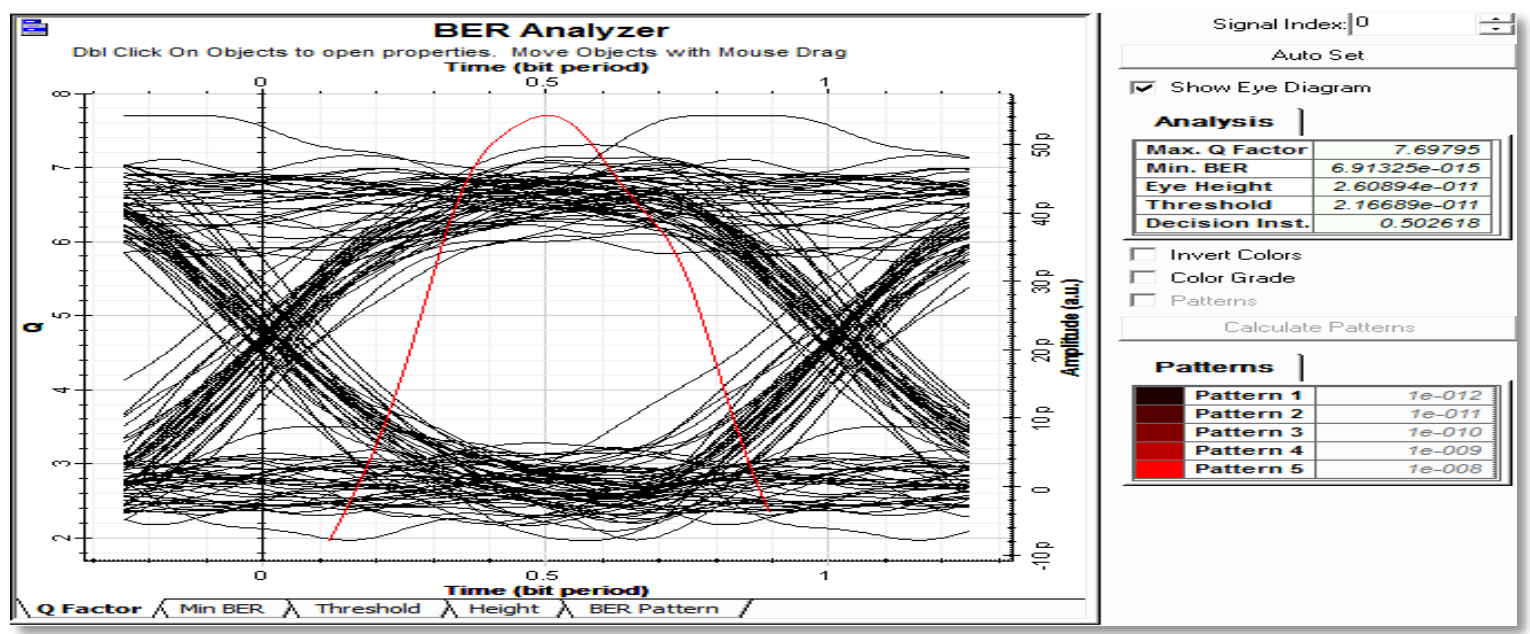

Figure 6 Eye diagram of max haze
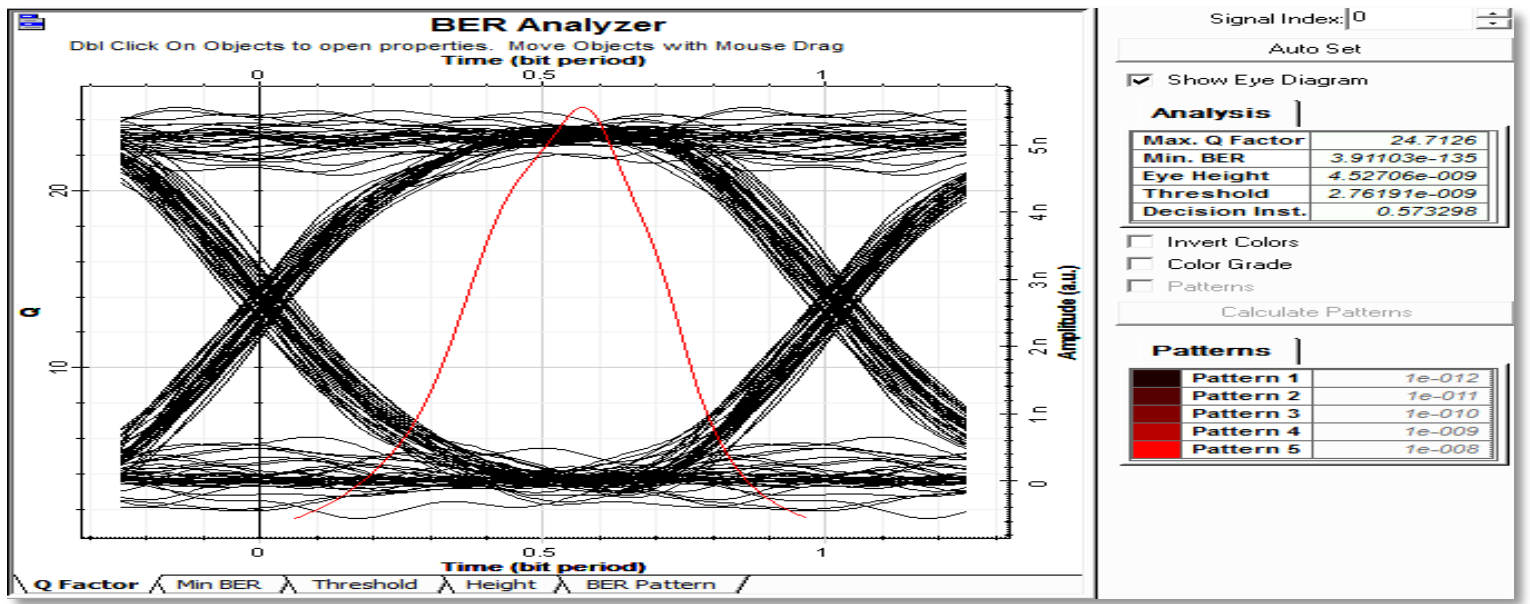

Figure 7 Eye diagram of mid haze 


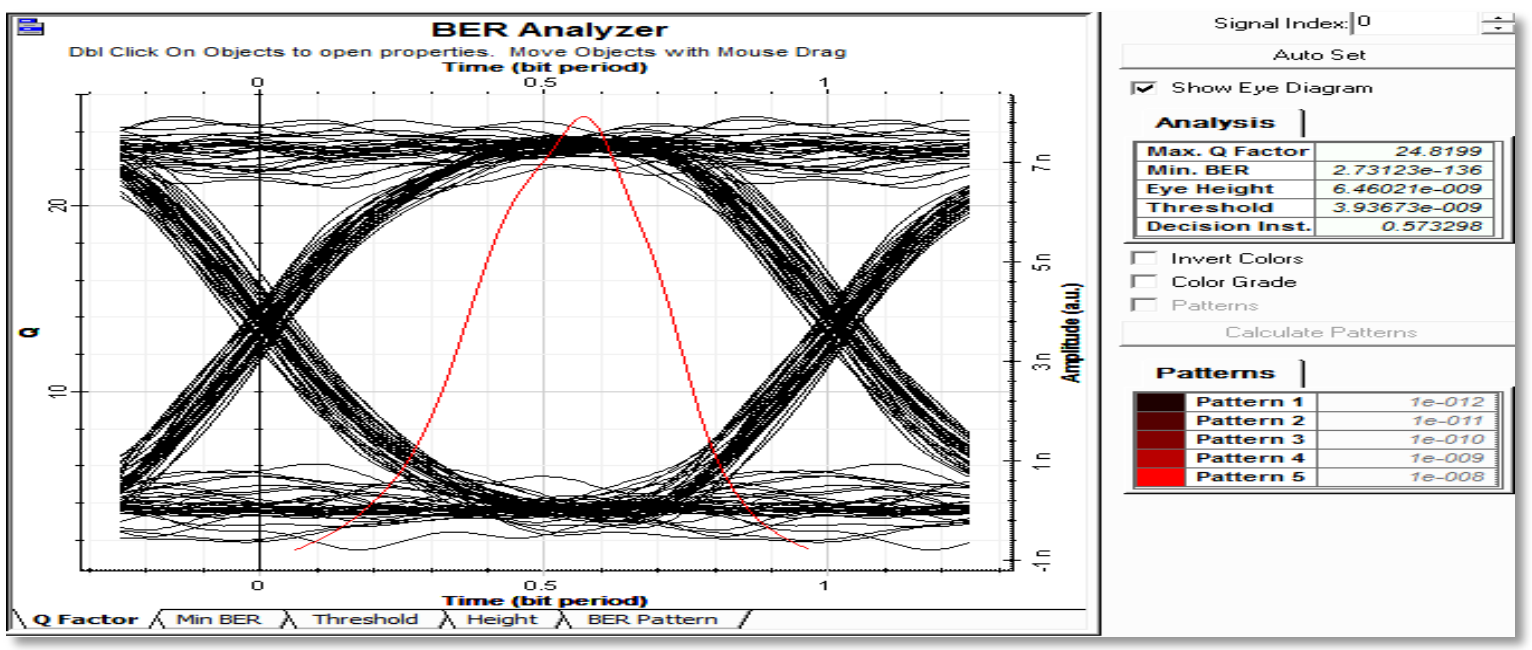

Figure 8 Eye diagram of light haze

\subsection{Experiment setup (Lab Scale)}

The equipment that used to execute the lab experiment to represent the FSO system and weather effect on the signal is:

1. Light source [laser led $(670 \mathrm{~nm}, 785 \mathrm{~nm})]$.

2. Chamber [glass room used to represent FSO concept; this chamber must have capability led to similar weather condition [rain, haze]. To perform rain state, the glass chamber should be equipped with a water sprayer controlled by an external water pump. To perform haze state, the glass room should contain a side fan to spread the soil in the rooms.

3. Ocean optic device [spectrometer device "small handheld instrument"] that has the capability to detect the received spectrum light and then translates it to the computer using USP connector. Then, ocean optic software in the computer draws the spectrum of the light [13].

Figure 9 represents how the equipment's arranged to form the complete FSO system. Laser led start to emit his laser beam through the glass room "chamber" which in turn perform the function of air effect. After reaching to the receiver side, ocean optics spectrum detector receives the light and internally translates it to the computer using the serial peripheral interface (SPI) connector. The ocean optic software reads the received signal and draws it by the software by figure that give relation between the intensity count of light and the type of wavelength that used in the experiment. The power of each laser led [without effect "control", rain effect, haze effect] is calculated using the following Equation [14]:

Power $(\mathrm{mw})=$ Intensity $\times$ Area
Where the intensity count of light can be noticed from the software and area can be calculated using Equation 8 .

Area $=\pi \times W_{O}^{2}$

Where $W_{o}$ is distance of any point on the surface. Table 4 shows the power of each laser and the absorption that occur due to [chamber effect]. It is clear that rain can largely attenuate the signal and absorbs the photon power.

Figures 10 show the ocean optic software results. The types of figures show the intensity agents the laser wavelength that used in the lab experiment. The FSO chamber can widely effects on the signal performance and leads to decrease the intensity count of laser light in the final receiver. In the figure below, system software records best result about the count of light because there is no external influence on the laser led.

The result in Figure 10 that shows the intensity count of light when the glass room "chamber" in a clear state. The signal travels through the room with no external signal effect like dust or rain particles. In this state signal can record the highest light intensity, especially in $785 \mathrm{~nm}$ because when system wavelength increases, the ray light phenomena decrease. This means that laser travel in a smooth path to the final point.

Figures 11 and 12 shows how the system glass room can work in the lab to present rain and haze state phenomena. The two types of laser $(785 \mathrm{~nm}$ and 670 $\mathrm{nm})$ in this state passes through water or dust particles and certainly the signals attenuate due to 
Jabar et al.

affected by the particles that separate in the glass room. When laser LED pass signal through the water particles, its loss its energy due to the absorption of the water molecules and the detector will record less light intensity count in the result. The dust also attenuates the signal by absorbing or scattered the light that hold the data, hence the intensity count also decrease.

Figures 13 and 14 shows the intensity count results for haze and rain effect, in which the glass room must separate dust or rain to represent rainy and haze weather phenomena. Figure 10, 13 and 14 shows the result of the lab experiment which gives the relation between the intensity count of light in the detector spot and the type of wavelength that used in the experiment. The intensity count record [in $670 \mathrm{~nm}$ $\lambda=1500$, in $785 \mathrm{~nm} \lambda=16400]$ in clear weather where no dust or rain particles separated through glass room. In rain weather state, the count decrease due to effect of rain particles on the signal laser and the software records intensity (in $670 \mathrm{~nm} \lambda=7000$ ) and (in $785 \mathrm{~nm} \lambda=9000$ ). In hazy weather, the count decreases (but not much higher than the rain state) due to effects of dust particles on the signal laser and the software record intensity (In $670 \mathrm{~nm} \lambda=12000$ ) and (In $785 \mathrm{~nm} \lambda=13000$ ). The results of the lab experiment ensure what comes in software part that rain effect on the FSO quality larger than haze phenomena.

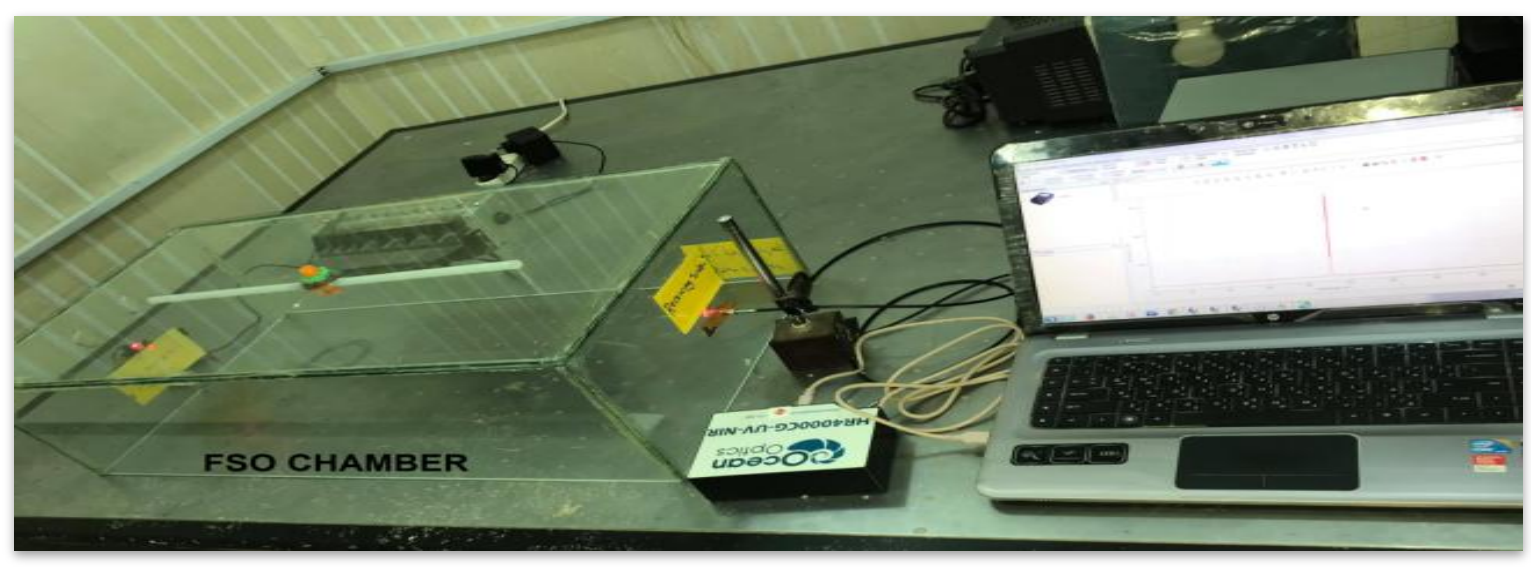

Figure $9 \mathrm{Lab}$ experiment device connected together to form FSO system

Table 4 The power for each state of laser and weather states

\begin{tabular}{c|cccccc}
\hline \multirow{2}{*}{ Wavelength } & \multicolumn{2}{|c}{$\begin{array}{c}\text { Control } \\
\text { without effect }\end{array}$} & \multicolumn{2}{c}{ With rain effect } & \multicolumn{2}{c}{ With haze effect } \\
\cline { 2 - 7 } & Power $(\mathbf{m w})$ & Power $(\mathbf{d B})$ & Power $(\mathbf{m w})$ & Power $(\mathbf{d B})$ & Power $(\mathbf{m w})$ & Power $(\mathbf{d B})$ \\
\hline $\mathbf{6 7 0} \mathbf{~ n m}$ & 4241.15 & 36 & 2261.9 & 33 & 3251.54 & 35.12 \\
\hline $\mathbf{7 8 5} \mathbf{~ n m}$ & 4806.63 & 38 & 2544.69 & 34 & 3817.03 & 35.769 \\
\hline
\end{tabular}
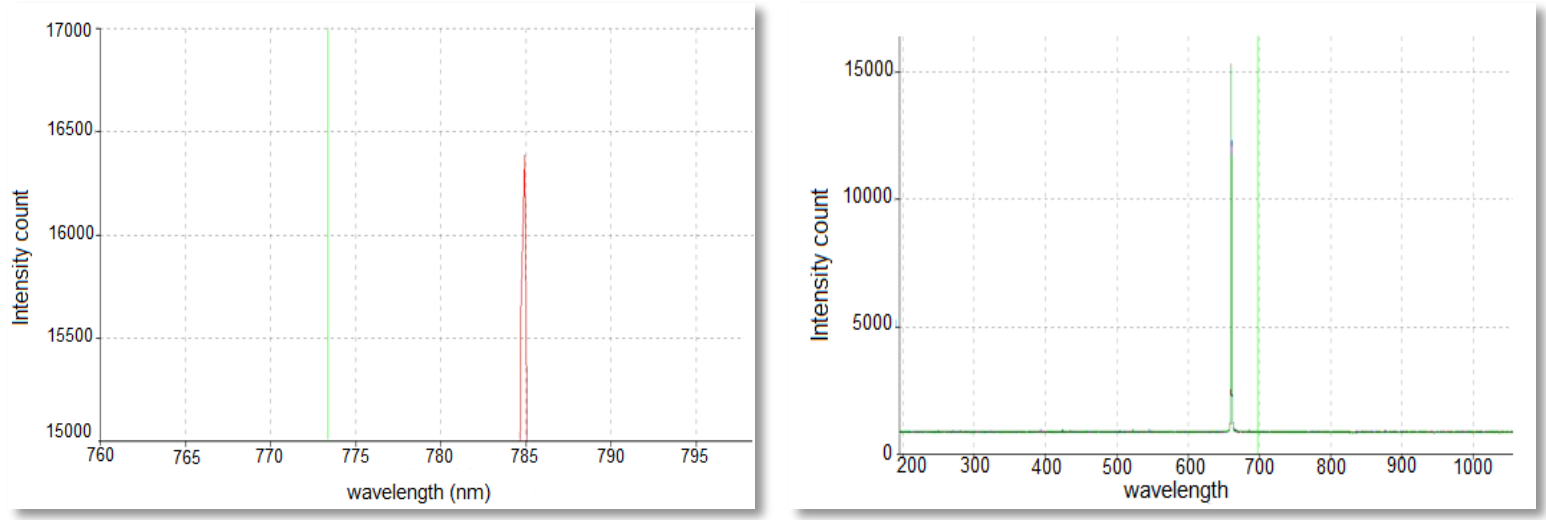

Figure 10 The intensity count of light when glass room in clear state (without any effect) 
International Journal of Advanced Computer Research, Vol 8(39)

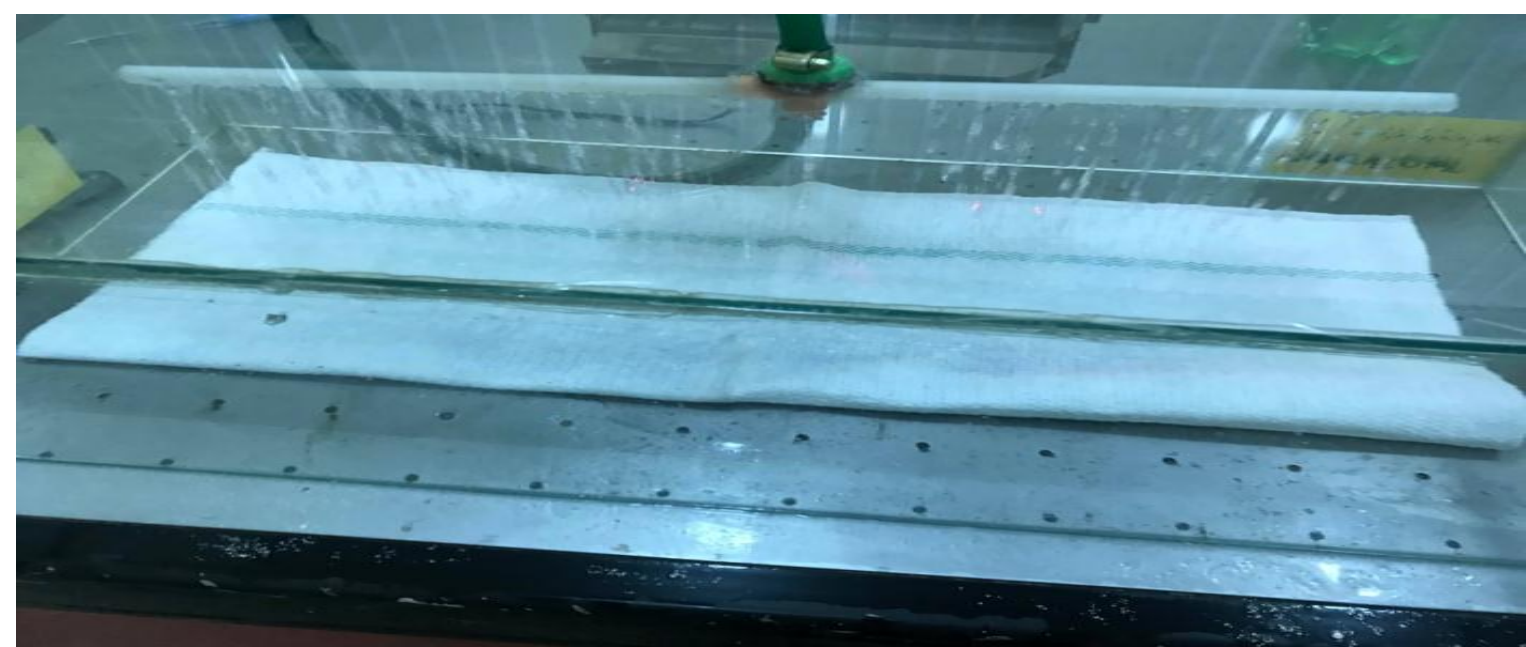

Figure 11 Behave of glass room in present of rain

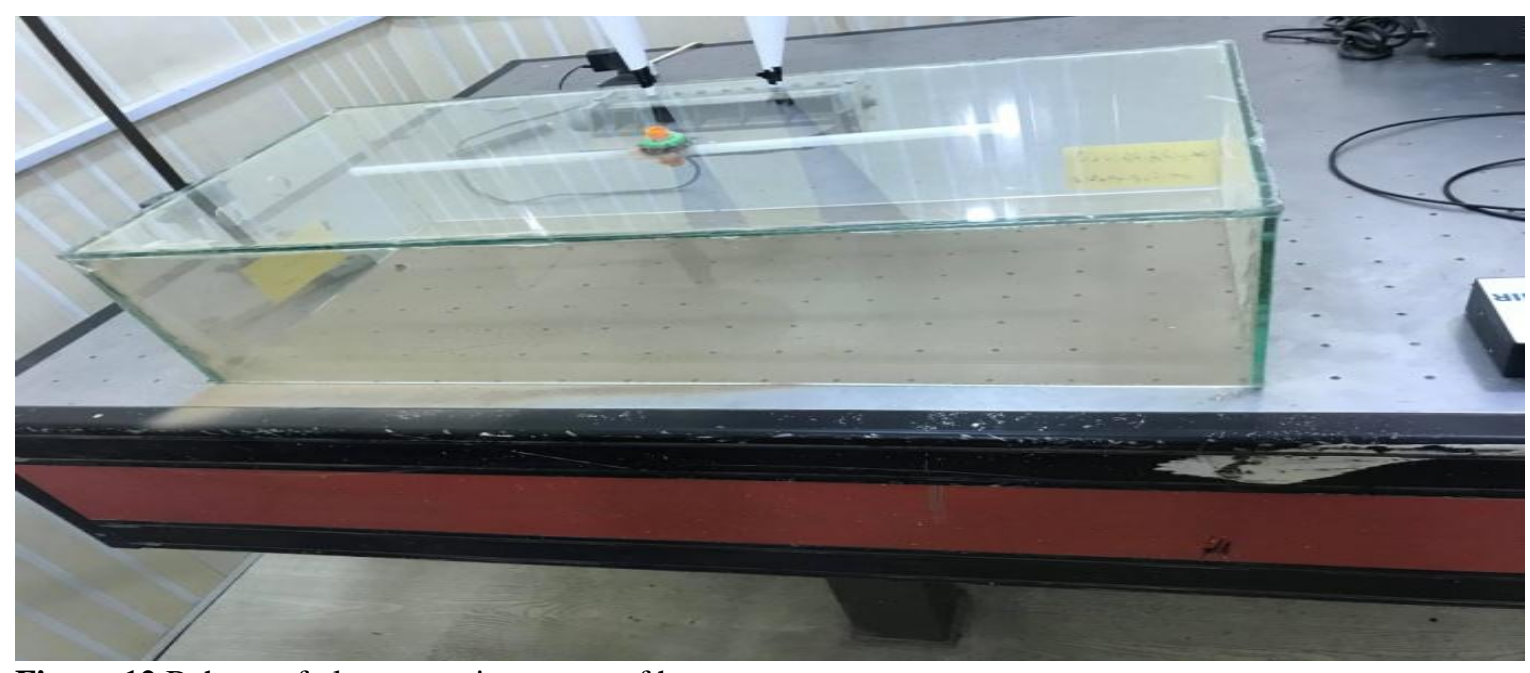

Figure 12 Behave of glass room in present of haze
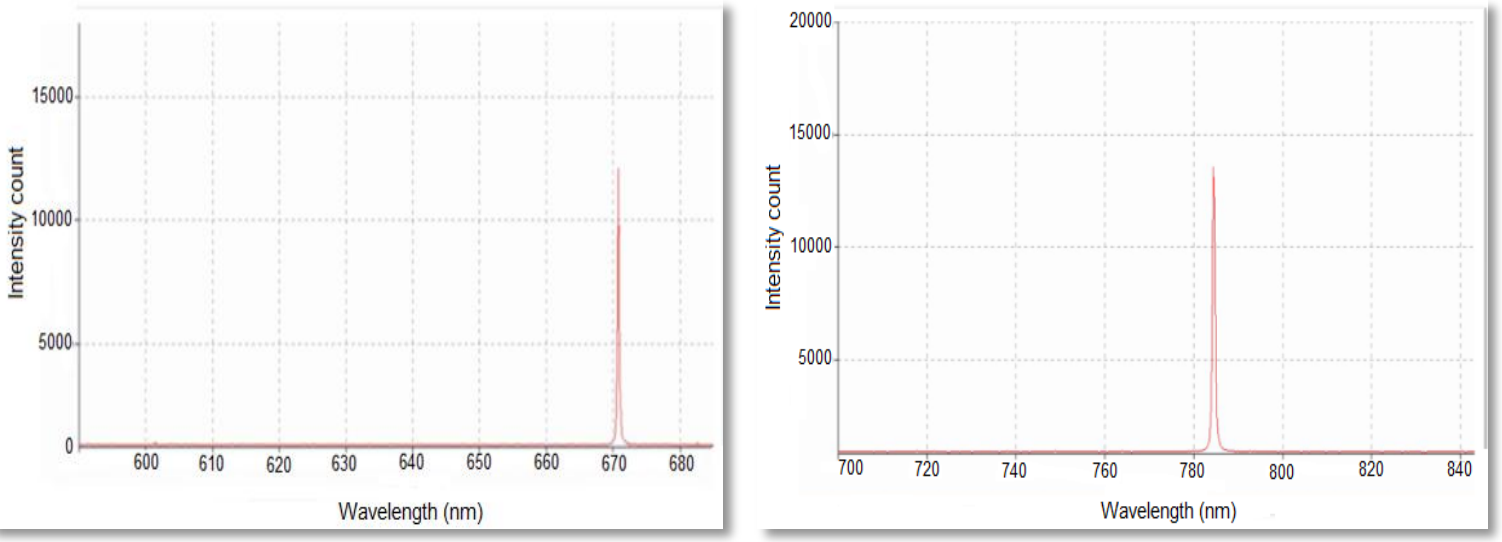

Figure 13 The wavelength agents' intensity count with haze effect on laser beam at $670 \mathrm{~nm}$ and $785 \mathrm{~nm}$ 
Jabar et al.
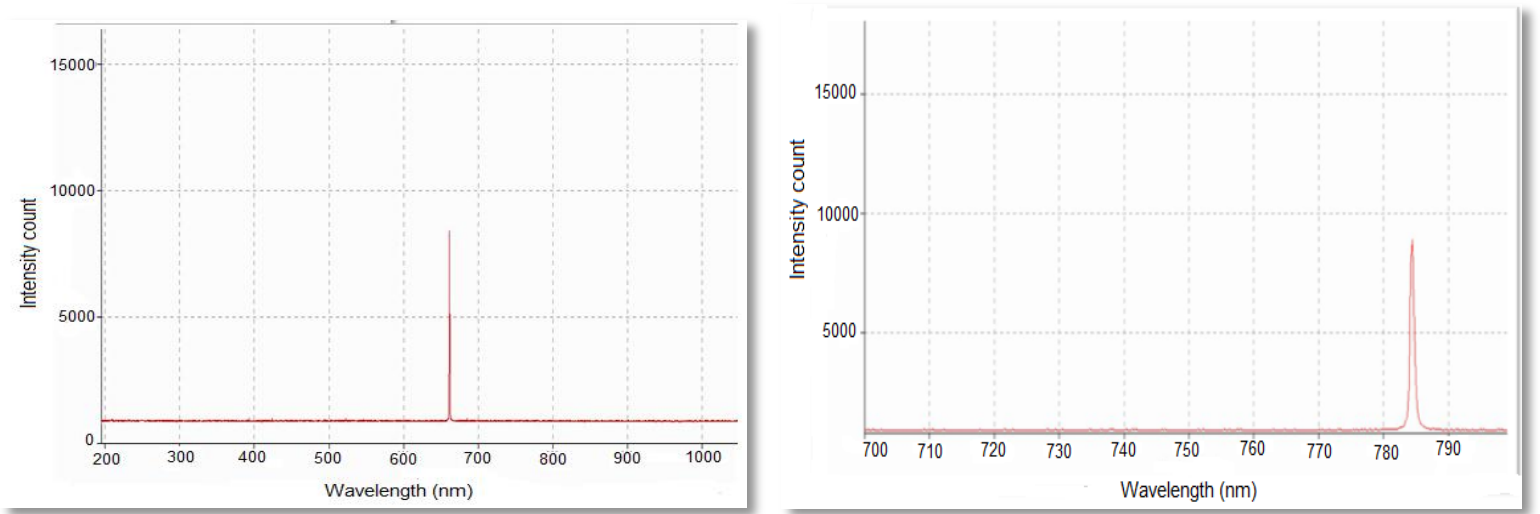

Figure 14 The wavelength agents' intensity count with rain effect on laser beam at $670 \mathrm{~nm}$ and $785 \mathrm{~nm}$

\section{Conclusion}

FSO technique is a good alternative technique, especially when compared with fiber cable. This study discussed the advantaged of using SCM-SACOCDMA with OA based on MD code and studies the effect of the weather phenomena [rain and haze] on signal parameter of FSO signal and how can reduce the effect of free space or multiple access interference (MAI) on the signal quality. SCM-SAC-OCDMA with MD code system and intermediate stage called "optical amplifier" used to permit the user data to travel through long distance and increase the number of users that share a single laser beam, while the MD code give the system zero cross correlation even when a large number of users used the same system. The rain effects on signal power more than haze particle effects. In rainy weather, signals can travel through $1.35 \mathrm{~km}$ as the transmission distance that the laser can cut it and can cut the $1.85 \mathrm{~km}$ as the transmission distance in haze weather state. The BER records $1 \times 10^{-14}$ under heavy rain and record $1 \times$ $10^{-15}$ under minimum visibility. It is clear that in haze weather signal can travel through long distance with minimum number of BER. This paper also shows a new lab experiment with the help of an ocean optic device to notice the weather effect on the laser by drawing the figure that indicates the relation between intensity count and the wavelength that used in the experiment. The practical parts ensure software results that rain attenuates the signal more than hazy weather. In rain state, intensity count of light decreases from 16400 to 9000 in $785 \mathrm{~nm}$ and decreases from 15000 to 8000 in $670 \mathrm{~nm}$ and in haze state, intensity count of light decreases from 16400 to 13500 in $785 \mathrm{~nm}$ and decreases from 15000 to 12000 in $670 \mathrm{~nm}$. Finally, to keep continues to enhance FSO technique, some future suggestion component added to the design of the system like automatic gain controller (AGC) or reflector.

\section{Acknowledgment}

None.

\section{Conflicts of interest}

The authors have no conflicts of interest to declare.

\section{References}

[1] Majumdar AK. Advanced free space optics (FSO): a systems approach. Springer; 2014.

[2] Sasaki K, Minato N, Ushikubo T, Arimoto Y. First OCDMA experimental demonstration over free space and optical fiber link. In conference on optical fiber communication/national fiber optic engineers conference, OFC/NFOEC. 2008 (pp. 1-3). IEEE.

[3] Arora S, Sharma A, Singh H. Performance analysis of multichannel OCDMA-FSO network under different pervasive conditions. In the proceedings of the international conference on communication and computing system 2017(pp.285-9). CRC Press.

[4] Ghafouri-Shiraz H, Karbassian MM. Optical CDMA networks: principles, analysis and applications. John Wiley \& Sons; 2012.

[5] Dutta NK, Wang Q. Semiconductor optical amplifiers. World Scientific; 2013.

[6] Willebrand H, Ghuman BS. Free space optics: enabling optical connectivity in today's networks. SAMS Publishing; 2002.

[7] Chan VW. Free-space optical communications. Journal of Lightwave Technology. 2006; 24(12):475062.

[8] IRAQ meteorological department, ministry of transparence report 2016.

[9] Kartalopoulos SV. Free space optical networks for ultra-broad band services. John Wiley \& Sons; 2011.

[10] Singh M, Bhatia S, Kaushal H. Performance evaluation of free space optical link under various weather conditions. In proceedings of the second international conference on computer and 
communication technologies 2016 (pp. 329-42). Springer.

[11] Gumaste A, Antony T. First mile access networks and enabling technologies. Cisco Press; 2004.

[12] Fayadh RA, Hamadi AM. Performance improvement of hybrid SCM SAC-OCDMA networks using multi diagonal code. Al-Ma'mon College Journal. 2016:33655.

[13] Tonkin SF. Practical amateur spectroscopy. Springer Science \& Business Media; 2013.

[14] An OER from textbook equity. College physics textbook equity. Createspace Independent Pub; 2012.

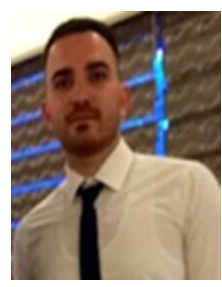

Hashem Jabar received his B.Sc. degree from Al-Mansour College (MUC) of Engineering / Iraq-Baghdad in 2013 and worked as an engineer in the Ministry of Water Resources until 2016. He is researching in order to get M.Sc. in Wireless Communication Systems for Design and Performance Analysis, with a special focus on FSO techniques to develop this type of communication in order to transmit high-altitude signals. Some revised journals and conference papers have been published.

Email: hashem.jabar@yahoo.com

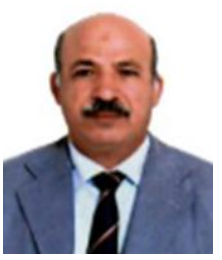

Rashid Ali Fayadh received his B.Sc. degree from Middlesex University /Engineering College / UK, in 1986 and worked as an Electronic Warfare Engineer in Iraqi air force up to 1998. He received the M.Sc. degree from University of Technology/ Al-Rasheed College/Baghdad-Iraq in 2000 . He was a lecturer from 2001 to 2012 in the Electrical and Electronic Technical College, Foundation of Technical Education, Baghdad, Iraq. The Ph.D. Degree in Wireless Communications at school of Computer and Communication Engineering, University Malaysia Perlis, Malaysia. $\mathrm{He}$ is doing research in the Design and Performance Analysis Wireless Communication Systems, with specific focus on Ultra-Wideband (UWB) technologies. He has published some reviewed journal and conference papers. Now I start work research wireless technique using the Optical FSO system.

Email: rashidfayadh@gmail.com

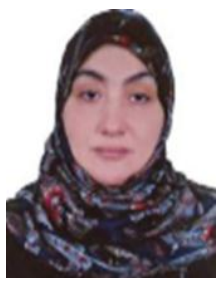

Mariam Mohamed Abud is teaching at the Mustansiriyah University, College of Education, Department of Physics. She worked as a lecturer in the Faculty of Science and Science for Girls in the Physics Department, University of Baghdad and is a patent from the standardization and Quality Control Department of patents and a member of the Iraqi Inventors Association. She has worked as a lecturer and lecturer since 1990 at the College of Science, Physics Department, University of Baghdad. She was appointed to the University of Basra College of Engineering Department of Materials Engineering (2006-2008).

Email: marmoh_1968@yahoo.com 